\title{
Downlink Coverage Probability in MIMO HetNets with Flexible Cell Selection
}

\author{
Abhishek K. Gupta, Harpreet S. Dhillon, Sriram Vishwanath, Jeffrey G. Andrews
}

\begin{abstract}
In this paper, we study the coverage probability of a $K$-tier multiple-input multiple-output heterogeneous cellular network (MIMO HetNet) assuming (i) zero-forcing precoding at all the base stations (BSs), (ii) Rayleigh fading, (iii) independent Poisson Point Process (PPP) model for the locations of BSs of each tier, and (iv) general cell selection rule that maximizes average received signal-to-interference-plus-noise ratio (SINR) at the users. Our analysis highlights key differences between MIMO HetNets and the more familiar single antenna HetNets in terms of cell selection. While it is challenging to derive exact cell selection rule to maximize average downlink SINR in MIMO HetNets, we show that adding an appropriately chosen per-tier selection bias yields a close approximation. The bias value for each tier is given in closed form. One interpretation of this result is that MIMO HetNets may balance load more naturally across different tiers in certain special cases compared to single antenna HetNets where an artificial selection bias is often needed for load balancing.
\end{abstract}

Index Terms-Heterogeneous cellular network, MIMO, stochastic geometry, cell selection bias, load balancing.

\section{INTRODUCTION}

Addition of various types of low power BSs, collectively termed as small cells, is continuously increasing the heterogeneity and irregularity of current cellular networks. With conventional cellular models, such as deterministic grid and Wyner model, becoming increasingly inaccurate, random spatial models have emerged as a promising new direction to model and analyze HetNets. In particular, modeling a cellular network as a superposition of independent PPPs, each modeling a particular BS type, such as macro, micro, pico and femtocells, lends tractability and provides easy-touse expressions for key performance metrics. This baseline model was introduced in [1], [2] and extended in [3]-[6] to study various aspects of HetNets. Please refer to [7] for a detailed survey. It is worth noting that most of the prior art in this line of work assumes single-input single-output (SISO) transmission. This is clearly an over-simplification of reality, where the coexistence of multi-antenna transmission and HetNets is almost inevitable.

Although the multi-antenna transmission techniques are well understood in isolation [8], their interplay with HetNets is an active area of research. Extending the PPP-based HetNet model of [2] to MIMO HetNets, an upper bound on coverage probability was derived in [9]. Tools from stochastic orders were used to order the performance of various multi-antenna transmission techniques for MIMO HetNets in [10]. On the

A. K. Gupta, J. G. Andrews and S. Vishwanath are with WNCG, The University of Texas at Austin. Email: g.kr.abhishek@utexas.edu, \{sriram, jandrews\}@ece.utexas.edu. H. S. Dhillon is with Wireless@VT, Department of ECE, Virginia Tech, Blacksburg, VA. Email: hdhillon@vt.edu. other hand, a hybrid MIMO HetNet model, which considers a fixed size "typical" cell was studied in [11]. Despite these efforts, the fundamentals of cell selection in MIMO HetNets, especially when different BSs adopt different multi-antenna transmission techniques and serve different number of users, are relatively unexplored. Our main focus is to initiate a systematic study towards understanding these fundamentals.

On the same lines as [9], [10], we assume $K$ different types of BSs, which may differ in terms of transmit power, deployment density, number of transmit antennas, transmission scheme, and number of users served. However, unlike [9], [10], where cell selection was implicitly based on maximum instantaneous signal-to-interference ratio (SIR), in this paper we focus on maximum average SINR-based cell selection. The key contributions are summarized below.

Cell selection: We show that the cell selection rules in MIMO HetNets are fundamentally different from those of SISO HetNets. For instance, in SISO HetNets, the BS that maximizes the downlink received power is also the one that maximizes the downlink SINR. However, this is not necessarily the case in MIMO HetNets due to precoding gain. We focus on maximum average received SINR-based cell selection and show that a simpler selection rule based on adding an appropriately chosen per-tier selection bias in the received power yields a surprisingly close approximation. We also derive this approximate per-tier selection bias in closed form. One key observation is that the bias value only depends upon the number of antennas at the BS and the number of users served in each resource block, which makes it easier to implement it in practice. Due to this connection with selection bias, MIMO HetNets have a more balanced load across tiers in certain regimes compared to SISO HetNets. To the best of our knowledge, this is the first systematic study that explores connections between cell selection and load balancing (across tiers) in the context of downlink MIMO HetNets.

Coverage probability: Assuming a general cell selection bias for each tier, an exact expression for coverage probability is also derived. After validating the expression through extensive system simulations, we use it to compare the performance of various transmission schemes in MIMO HetNets.

\section{SySTEM MODEL}

We consider a HetNet consisting of BSs of different classes, which may differ in terms of transmit power $P_{k}$ (to each user), deployment density $\lambda_{k}$, number of transmit antennas $M_{k}$, transmission scheme, and number of users served in each resource block $\Psi_{k}$. We assume that there are $K$ different 
classes or tiers of BSs indexed by the set $\mathcal{K}=\{1,2, \ldots K\}$. The locations of each tier are sampled from an independent homogeneous PPP $\Phi_{k}$ of density $\lambda_{k}$ which has been justified both by empirical evidence [12] and theoretical validation [13]. The overall point process consisting of all BSs' locations is represented by the superposition of $K$ PPPs as $\Phi=\cup_{k \in \mathcal{K}} \Phi_{k}$. A particular realization of $\Phi$ will be denoted by $\phi$. The users are assumed to form an independent PPP $\Phi_{u}$ of density $\lambda_{u}$. Each user is assumed to have a single receive antenna. The analysis is performed at a typical user located at the origin.

In this paper, we will restrict our attention to zero-forcing precoding, which is general enough to encompass important transmission schemes such as beamforming and spatial division multiple access (SDMA), while being tractable enough to provide important system design guidelines. Note that due to precoding at the BS, the effective channel gain to a given receiver depends upon whether that BS acts as a serving BS or an interferer for that receiver. For example, if a multi-antenna transmitter beamforms to a given user, the effective channel gain would be much higher than when it simply acts as an interferer. Therefore, we denote the effective channel power gain from a $k^{t h}$ tier BS located at $x_{k} \in \Phi_{k}$ to a typical user by $h_{x_{k} k}$ when it acts as a serving BS, and by $g_{x_{k} k}$ when it acts as an interferer. We also assume perfect channel state information (CSI) at the transmitter, although as argued in [10] the tools developed in this paper can also be used to incorporate the effects of imperfect CSI. For zero-forcing precoding with perfect CSI under Rayleigh fading, we can approximate $h_{x_{k} k} \sim \Gamma\left(\Delta_{k}, 1\right)$ and $g_{x_{k} k} \sim \Gamma\left(\Psi_{k}, 1\right)$, where $\Delta_{i}=M_{i}-\Psi_{i}+1$ as discussed in [10]. The received power at a typical user from a serving BS located at $x_{k} \in \Phi_{k}$ is

$$
P\left(x_{k}\right)=P_{k} h_{x_{k} k}\left\|x_{k}\right\|^{-\alpha},
$$

where $\left\|x_{k}\right\|^{-\alpha}$ is a standard power-law path-loss with exponent $\alpha$ and $P_{k}$ is the transmit power of the $k^{t h}$ tier BS. The resulting $\operatorname{SINR} \gamma_{k}\left(x_{k}\right)$ is

$$
\gamma_{k}\left(x_{k}\right)=\frac{P_{k} h_{x_{k} k}\left\|x_{k}\right\|^{-\alpha}}{I+N},
$$

where $N$ is the noise power, and $I$ is the interference power given by

$$
I=\sum_{j \in \mathcal{K}} I_{j}=\sum_{j \in \mathcal{K}} \sum_{x \in \Phi \backslash x_{k}} P_{j} g_{x j}\|x\|^{-\alpha} .
$$

For brevity, we will assume the interference limited network where noise power $N=0$. For cleaner exposition in the next section, let us denote the average received power from a $k^{t h}$ tier BS by $P_{r k}\left(x_{k}\right)$ given as

$$
P_{r k}\left(x_{k}\right)=P_{k} \Delta_{k}\left\|x_{k}\right\|^{-\alpha} .
$$

We now study the fundamentals of cell selection for MIMO HetNets in the next section.

\section{Cell Selection in Mimo HetNets}

Recall that a usual cell selection criterion in SISO HetNets is to select the BS that provides the maximum average received power, possibly with a certain bias value for load balancing.
In the case when there is no bias, this cell selection criterion is also the one that maximizes SINR. Therefore, to maximize coverage probability, a typical user simply connects to the BS that provides the highest received power, as discussed in [3]. However, it is easy to construct a simple toy example showing that this is not the case in MIMO HetNets.

Example 1. Consider two BSs at the same distance from a typical user, one having 4 antennas serving a single user, which with a slight overloading of the notation implies $M_{1}=$ $4, \Psi_{1}=1, \Delta_{1}=4$, and the other serving 4 users with 6 antennas, i.e., $M_{2}=6, \Psi_{2}=4, \Delta_{2}=3$. Since $\Delta_{1}>\Delta_{2}$, a typical user should connect to the first $B S$ to maximize average received power. However, since $\Psi_{2}>\Psi_{1}$, the interference from second BS is larger than the first and it should connect to second to minimize the received interference power. Further, since $\frac{\Delta_{2}}{\Psi_{1}}>\frac{\Delta_{1}}{\Psi_{2}}$, it should connect to the second $B S$ to maximize its received SINR.

We first discuss cell selection with the goal of maximizing the average received SINR conditional on the point process $\Phi$. Note that to maximize both the average received power and average SINR, it is strictly suboptimal for a typical user to connect to any BS except the ones that are closest to it in each tier, i.e., a typical user should only connect to one of BSs from the set $S=\left\{x_{i}, i \in \mathcal{K}: x_{i}\right.$ is the closest BS of tier $\left.i\right\}$. Let $\gamma_{k}$ be the instantaneous SINR when a typical user connects to the closest $k^{\text {th }}$ tier BS. Under maximum average SINR cell selection rule, $k^{\text {th }}$ tier is selected if

$$
k=\arg \max _{j \in \mathcal{K}} \mathbb{E}\left[\gamma_{j} \mid \Phi=\phi\right],
$$

where the selection rule clearly depends upon the distances of the BSs to a typical mobile due to conditioning on the realization of the point process $\Phi$. Since it is quite challenging to derive an exact selection rule from (5), we will present an approximate but simple and practical cell selection rule that works across wide range of system parameters using Jensen's inequality.

In a given realization $\phi$ of point process $\Phi$, let us assume that there are two possible candidate serving BSs: $k^{\text {th }}$ tier BS located at $x_{k}$ and $j^{\text {th }}$ tier BS located at $x_{j}$. Let $W$ denote the residual interference from other BSs and $a_{i}=P_{i}\left\|x_{i}\right\|^{-\alpha}$. Given this realization, the average SINR is given by

$$
\begin{aligned}
\mathbb{E}\left[\gamma_{k}\left(x_{k}\right) \mid \phi\right] & =\mathbb{E}\left[\frac{h_{x_{k} k} a_{k}}{g_{x_{j} j} a_{j}+W} \mid \phi\right] \\
& =a_{k} \Delta_{k} \mathbb{E}\left[\frac{1}{g_{x_{j} j} a_{j}+W} \mid \phi\right] \\
& \geq a_{k} \Delta_{k} \frac{1}{\mathbb{E}\left[g_{x_{j} j} a_{j}+W \mid \phi\right]}=\frac{a_{k} \Delta_{k}}{\Psi_{j} a_{j}+\mathbb{E}[W \mid \phi]} .
\end{aligned}
$$

Using this lower bound, the conditions required for selection of $x_{k}$ over $x_{j}$ are

$$
\begin{aligned}
& P_{k} \Delta_{k}\left\|x_{k}\right\|^{-\alpha} \geq P_{i} \Delta_{i}\left\|x_{i}\right\|^{-\alpha_{i}} \quad \forall i \neq k \\
& P_{k} \Psi_{k}\left\|x_{k}\right\|^{-\alpha} \geq P_{i} \Psi_{i}\left\|x_{i}\right\|^{-\alpha_{i}} \quad \forall i \neq k,
\end{aligned}
$$

which need to be satisfied simultaneously. The following example demonstrates that this is not always possible, i.e., none of the tiers may satisfy both these conditions simultaneously. 
Example 2. Consider a two tier network such that the distance of the typical user to the nearest BS in each tier is the same, i.e., $x_{1}=x_{2}$. Further assume that the transmit powers for the two tiers are also the same. Fixing $\Delta_{1}=2, \Delta_{2}=1, \Psi_{1}=$ $2, \Psi_{2}=3$, it is easy to check that neither $k=1$ nor $k=2$ satisfy (6) and (7) simultaneously.

To construct a condition which guarantees a solution, we multiply the two inequalities to get a simpler but more useful sufficient condition for the selection of $k^{\text {th }}$ tier

$$
P_{k} \sqrt{\Delta_{k} \Psi_{k}}\left\|x_{k}\right\|^{-\alpha} \geq P_{i} \sqrt{\Delta_{i} \Psi_{i}}\left\|x_{i}\right\|^{-\alpha} \quad \forall i \neq k .
$$

It is easy to verify that there always exists a $k \in \mathcal{K}$ for which the selection law (8) holds and is equal to the solution of (6) and (7) if the solution of the latter pair exists. The following remark presents an interesting connection between this cell selection criterion and idea of cell selection bias.

Remark 1 (Connections with biasing). We can rewrite

$$
\sqrt{\frac{\Psi_{k}}{\Delta_{k}}} P_{r k}\left(x_{k}\right) \geq \sqrt{\frac{\Psi_{i}}{\Delta_{i}}} P_{r i}\left(x_{i}\right) \quad \forall i \neq k,
$$

where $P_{r k}\left(x_{k}\right)=P_{k} \Delta_{k} x_{k}^{-\alpha}$ is the average received power from a $k^{\text {th }}$ tier BS located at $x_{k} \in \Phi_{k}$. Here $B_{k}=\sqrt{\frac{\Psi_{k}}{\Delta_{k}}}$ can be perceived as a "bias". For SISO case, $\Psi_{k}=\Delta_{k}=1 \forall k \in$ $\mathcal{K}$, and the above rule reduces to selecting the $B S$ providing highest received power, which is also the SINR maximizing rule for the SISO case.

Similar to (8), other candidate laws can also be derived by different way of combining, e.g., by adding the inequalities (6) and (7), which gives:

$$
P_{k}\left(\Delta_{k}+\Psi_{k}\right) x_{k}^{-\alpha} \geq P_{i}\left(\Delta_{i}+\Psi_{i}\right) x_{i}^{-\alpha} \quad \forall i \neq k,
$$

which can also be interpreted as a selection bias with $B_{k}=1+$ $\frac{\Psi_{k}}{\Delta_{k}}$ in the context of Remark 1. We evaluate the approximation error for these cell-selection laws in numerical result section and show that the selection bias of $B_{k}=\sqrt{\frac{\Psi_{k}}{\Delta_{k}}}$ is surprisingly accurate for SINR, and hence coverage, maximization. Note, however, that this choice of selection bias is not necessarily optimal for other metrics, e.g., rate coverage. Therefore, to maintain generality, we derive all the results in terms of a general cell-selection bias term $B_{k}$, i.e., a user selects a nearest BS of $k^{t h}$ tier if

$$
k=\arg \max _{j \in \mathcal{K}} B_{j} P_{j} \Delta_{j}\left\|x_{j}\right\|^{-\alpha}
$$

or in other words, a typical user selects a BS that provides the maximum biased received power, where the bias values are tuned according to the metric that is being maximized which results in exclusion regions around a typical user and all the interfering BSs lie outside of this region. This exclusion region is formally defined in the following lemma.

Lemma 1. A $k^{\text {th }}$ tier BS situated at distance $R_{k}$ from the typical user located at the origin is selected if the distance of the typical user from all the other $B S s, R_{j}, j \neq k$, satisfies the following condition

$$
R_{j} \geq\left(\widehat{P}_{j} \widehat{B}_{j} \widehat{\Delta}_{j}\right)^{\frac{1}{\alpha}} R_{k}
$$

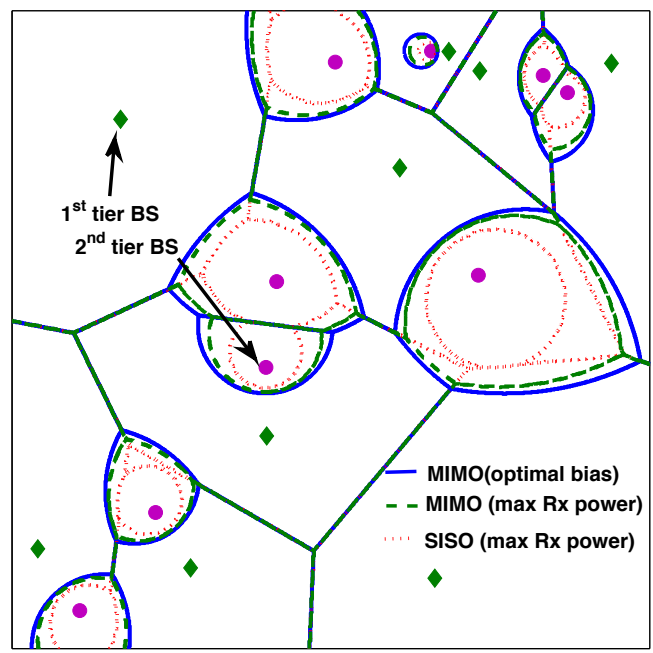

Fig. 1. The expansion of coverage regions due to inherent biasing in two tier MIMO HetNet with $\Psi=[1,6], P=[30,1], \Delta=[1,3]$. In this hypothetical case, small cells (denoted by filled circle) use 8 antennas and schedule 3 users per resource block. Macrocell BSs (denoted by diamonds) have single antenna per BS. Here, optimal bias refers to the bias that maximizes the mean SINR.

where $\widehat{P}_{j}=P_{j} / P_{k}$. The $\widehat{B}_{j}$ and $\widehat{\Delta}_{j}$ are defined similarly.

As discussed earlier in [2] for SISO HetNets, the association regions thus formed are weighted Voronoi regions. However, unlike SISO HetNets, the weights are not just equal to the received power but also include a bias term which accounts for minimizing the interference. We compare the association regions for SISO and MIMO HetNets for a fixed realization of the BS point process in Fig. 1 for two different cell selection rules: i) based on maximum received power, ii) based on maximum average SINR. For a SISO HetNet, both these selection rules are exactly the same. For a MIMO HetNet, the second selection rule may expand or shrink the association region compared to a SISO HetNet and hence may help in balancing load across tiers.

Before concluding this section, we state the cell selection (association) probability result, i.e., the probability with which a typical user selects a $k^{t h}$ tier BS, which will be useful in the coverage probability analysis in the next section. Using [3, Lemma 1], the cell selection probability is

$$
A_{k}=\frac{\lambda_{k}}{\sum_{j} \lambda_{j}\left(\widehat{P}_{j} \widehat{\Delta}_{j} \widehat{B}_{j}\right)^{\frac{2}{\alpha}}} .
$$

\section{Coverage Probability}

A typical user is said to be in coverage if its serving BS provides downlink SINR above a certain coding and modulation specific threshold $T$. It is formally defined as

$$
P_{c}=\sum_{k=1}^{K} \mathbb{P}\left[\gamma_{k}>T, k=\text { associated tier }\right] .
$$

For the mean SINR association model, the coverage probability is given as

$$
P_{c}=\sum_{k=1}^{K} \mathbb{E}_{\Phi_{k}}\left[\mathbb{1}_{\left(\gamma\left(x_{k}\right)>T\right)} \mathbb{1}_{\left(S_{x_{k}, \phi} \geq S_{x, \phi} \quad \forall x \in \phi \backslash x_{k}\right)}\right]
$$


where $\gamma_{k}$ and $\mathbb{E}\left[\gamma_{k} \mid \phi\right]$ are instantaneous SINR and conditional mean SINR given realization $\phi$ and $S_{x, \phi}=\mathbb{E}[\gamma(x) \mid \phi]$.

For the selection rule (12), the latter term (association indicator) can be expressed as

$$
e_{k}\left(x_{k}, \phi \backslash x_{k}\right)=\mathbb{1}\left(\left\|x_{i}\right\| \geq\left(\widehat{P}_{j} \widehat{\Delta}_{j} \widehat{B}_{j}\right)^{\frac{1}{\alpha}}\left\|x_{k}\right\| \forall x_{i} \in \phi \backslash x_{k}\right)
$$

and the probability of coverage can be computed as

$$
\begin{aligned}
P_{c} \stackrel{(a)}{=} & \sum_{k=1}^{K} \lambda_{k} \int_{0}^{\infty} \mathbb{P}\left(\frac{P_{k} h_{x}}{\|x\|^{\alpha}}>T\left(I_{\Phi}\right) \mid e_{k}(x, \phi)\right) . \\
& \mathbb{P}\left(\left\|x_{i}\right\| \geq\left(\widehat{P}_{j} \widehat{\Delta}_{j} \widehat{B}_{j}\right)^{\frac{1}{\alpha}}\|x\| \forall x_{i} \in \Phi\right) \mathrm{d} x \\
\stackrel{(b)}{=} & 2 \pi \sum_{k=1}^{K} \lambda_{k} \int_{0}^{\infty} \mathbb{P}\left(P_{k} h_{x}\|x\|^{-\alpha}>T\left(I_{\Phi^{\prime}}\right)\right) . \\
& e^{-\sum_{j=1}^{K} \lambda_{j} \pi\left(\widehat{P}_{j} \widehat{\Delta}_{j} \widehat{B}_{j}\right)^{\frac{2}{\alpha}} x^{2}} x \mathrm{~d} x,
\end{aligned}
$$

where $\Phi^{\prime}$ is given as $\Phi^{\prime}=\cup_{k=1}^{K} \Phi_{k} \cap \mathbb{B}\left(0,\left(\widehat{P}_{j} \widehat{\Delta}_{j} \widehat{B}_{j}\right)^{\frac{1}{\alpha}}\|x\|\right)^{c}$ where $\mathbb{B}(0, r)$ is open ball around origin with radius $r$ and $(.)^{c}$ denotes the set complement and signifies that all the interfering BSs are outside this ball. Here $(a)$ is from conditional probability and $(b)$ is due to property of a Poisson point process.

The probability term inside the integral in (16) represents CCDF of the SINR at typical user associated with the $k^{\text {th }}$ tier BS situated at a distance $x_{k}=x$. It can be expressed as

$$
\begin{aligned}
\mathbb{P}\left[\gamma_{k}(x)>T\right] & =\mathbb{P}\left[P_{k} h_{k} x^{-\alpha}>T(I)\right]=\mathbb{P}\left[h_{k}>\right. \\
& =\sum_{n=0}^{\Delta_{k}-1} \frac{1}{n !} \mathbb{E}\left[(-s(I))^{n} e^{-s(I)}\right] \\
& =\sum_{n=0}^{\Delta_{k}-1} \frac{1}{n !}(-s)^{i} \frac{\mathrm{d}^{n}}{\mathrm{~d} s^{n}}\left(\mathbb{E}\left[e^{-s(I)}\right]\right)
\end{aligned}
$$

with $s=T P_{k}^{-1} x^{\alpha}$.

To simplify (17) further, we will not only need the Laplace transform (LT) of interference but also the derivatives of LT.

\section{A. Laplace transform of Interference}

Lemma 2. The Laplace transform of interference from all the BSs at a typical user served by a $k^{\text {th }}$ tier BS located at a distance $x$ from the typical user is given by

$$
\begin{aligned}
\mathcal{L}_{I}(s) & =\exp \left[-2 \pi \sum_{j=1}^{K} \frac{\lambda_{j}}{\alpha}\left(s P_{j}\right)^{\frac{2}{\alpha}} \sum_{m=1}^{\Psi_{j}}\left(\begin{array}{c}
\Psi_{j} \\
m
\end{array}\right)\right. \\
& \left.B^{\prime}\left(\Psi_{j}+m-\frac{2}{\alpha}, m+\frac{2}{\alpha}, \frac{1}{1+\frac{s P_{j} x^{-\alpha}}{\left(\widehat{P}_{j} \widehat{\Delta}_{j} \widehat{B}_{j}\right)}}\right)\right]
\end{aligned}
$$

where $B^{\prime}(a, b, c)$ is the complementary incomplete Beta function defined as

$$
B^{\prime}(a, b, z)=\int_{z}^{1} u^{a-1}(1-u)^{b-1} d u .
$$

Proof: Due to limited space, we skip the proof. It follows mainly from Lemma 4 of [10] along with the tools developed in [3].

\section{B. Derivatives of the Laplace Transform}

The derivative of the LT is given by the following Lemma.

Lemma 3. The $n^{\text {th }}$ derivative of the Laplace transform of interference is $\frac{\mathrm{d}^{n}}{\mathrm{~d} s^{n}} \mathcal{L}_{I}(s)=$

$$
\mathcal{L}_{I}(s) \sum_{\bar{m} \in M} C(\bar{m}) \cdot \prod_{l=1}^{n}\left(2 \pi \sum_{j=1}^{K}(-1)^{l} D_{j}(l) P_{j}^{l}\left(s P_{j}\right)^{\frac{2}{\alpha}-l}\right)^{m_{l}}
$$

where

$$
\begin{aligned}
M(n) & =\left\{\bar{m}=\left(m_{1}, m_{2}, \cdots m_{n}\right)^{T}: \sum_{i=1}^{n} i m_{i}=n\right\} \\
C(\bar{m}) & =\frac{n !}{\prod_{i}\left(m_{i} !(i !)^{m_{i}}\right)} \\
D_{j}(l) & =\frac{\lambda_{j}}{\alpha} \frac{\left(\Psi_{j}+l-1\right) !}{\left(\Psi_{j}-1\right) !} B^{\prime}\left(\Psi_{j}+\frac{2}{\alpha}, l-\frac{2}{\alpha}, u_{j}\right) \\
u_{j} & =\frac{1}{1+\frac{s P_{j} x^{-\alpha}}{\left(\widehat{P}_{j} \widehat{\Delta}_{j} \widehat{B}_{j}\right)}} .
\end{aligned}
$$

Proof: The proof is based on the tools developed in [10]. See Appendix A for a sketch.

For the later use, we give the values of Laplace transform and its derivative at $s=T P_{k}^{-1} x^{\alpha}$ here:

- $\mathcal{L}_{I}\left(T P_{k}^{-1} x^{\alpha}\right)=e^{-T P_{k}^{-1} N x^{\alpha}-\left[\sum_{j=1}^{K} \lambda_{j}\left(T \widehat{P}_{j}\right)^{\frac{2}{\alpha}} \mathcal{C}_{j}\right] x^{2}}$ where $\mathcal{C}_{j}$ is defined as $\mathcal{C}_{j} \triangleq$

$$
\begin{gathered}
\frac{2 \pi}{\alpha} \sum_{m=1}^{\Psi_{j}}\left(\begin{array}{c}
\Psi_{j} \\
m
\end{array}\right) B^{\prime}\left(\Psi_{j}+m-\frac{2}{\alpha}, m+\frac{2}{\alpha}, \frac{1}{1+\frac{T}{\widehat{\Delta}_{j} \widehat{B}_{j}}}\right) \\
\text { - }\left.\frac{\mathrm{d}^{n} \mathcal{L}_{I}(s)}{\mathrm{d} s^{n}}\right|_{s=T P_{k}^{-1} x^{\alpha}}=e^{-\left[\sum_{j=1}^{K} \lambda_{j}\left(T \widehat{P}_{j}\right)^{\frac{2}{\alpha}} \mathcal{C}_{j}\right] x^{2}} \cdot \\
\sum_{\bar{m} \in M} C(\bar{m}) x^{-n \alpha+2 \sum m_{l}}(-1)^{n} F(\bar{m})
\end{gathered}
$$

where

$$
F(\bar{m}) \triangleq \frac{(2 \pi)^{\sum m_{l}}}{\left(T P_{k}^{-1}\right)^{-\frac{2}{\alpha} \sum m_{l}+n}} \prod_{l=1}^{n}\left(\sum_{j=1}^{K} D_{j}(l) P_{j}^{\frac{2}{\alpha}}\right)^{m_{l}}
$$

with $u_{j}=\frac{1}{1+\frac{T}{\widehat{\Delta}_{j} \widehat{B}_{j}}}$.

\section{SINR distribution and Coverage Probability}

We can now compute the CCDF of SINR using the results of previous subsections and equation (17).

Theorem 1. The CCDF of SINR at the typical user associated with the $k^{t h}$ tier BS situated at a distance $X_{k}=x$ from the typical user for the interference limited case is given as

$$
\begin{aligned}
\mathbb{P}\left[\gamma_{k}(x)>T\right]= & \sum_{n=0}^{\Delta_{k}-1} \frac{1}{n !}\left(T P_{k}^{-1}\right)^{n} \sum_{\bar{m} \in M} C(\bar{m}) F(\bar{m}) . \\
& e^{-\left[\sum_{j=1}^{K} \lambda_{j}\left(T \widehat{P}_{j}\right)^{\frac{2}{\alpha}} \mathcal{C}_{j}\right] x^{2}} x^{2 \sum m_{l}}
\end{aligned}
$$

with $u_{j}=\frac{1}{1+\frac{T}{\widehat{\Delta}_{j} \hat{B}_{j}}}$. 
Finally, we now compute the coverage probability of a typical user in a MIMO HetNet using (16).

Theorem 2. Coverage probability of a typical user in the interference limited case is given by $P_{c}(T)=$

$$
\begin{aligned}
& \sum_{k=1}^{K} \pi \lambda_{k} \sum_{n=0}^{\Delta_{k}-1} \frac{1}{n !}\left(T P_{k}^{-1}\right)^{n} . \\
& \sum_{\bar{m} \in M} \frac{C(\bar{m}) F(\bar{m}) \Gamma\left(\sum m_{l}+1\right)}{\left[\sum_{j=1}^{K} \lambda_{j}\left(T \widehat{P}_{j}\right)^{\frac{2}{\alpha}} \mathcal{C}_{j}+\pi \sum_{j=1}^{K} \lambda_{j}\left(\widehat{P}_{j} \widehat{\Delta}_{j} \widehat{B}_{j}\right)^{\frac{2}{\alpha}}\right]^{\sum m_{l}+1}}
\end{aligned}
$$

Proof: See Appendix B.

\section{Numerical Results}

In this section we present numerical results to validate our analysis and provide key system design insights. For simulations, $K$ independent Poisson point processes are generated over a large spatial window and selection of a BS and tier is done according to (11). The fading link from serving BS to a user is modeled as random variable $h_{i x} \sim \Gamma\left(\Delta_{i}, 1\right)$ and the interfering links are modeled as $g_{j x} \sim \Gamma\left(\Psi_{j}, 1\right)$. For brevity, we consider an interference limited two tier HetNet with macrocells forming the first tier and femtocells forming the second with $\lambda_{1}=150$ and $\lambda_{2}=300$. The following two multi-antenna techniques are considered for numerical results: (i) single-user beamforming (SUBF), where each BS serves single user, i.e., $\Delta_{i}=M_{i}, \Psi_{i}=1$, and (ii) full SDMA, where an $i^{\text {th }}$ tier BS serves $M_{i}$ users in each resource block, i.e., $\Delta_{i}=1, \Psi_{i}=M_{i}$. Combining these techniques with different system configurations, we define the following 7 simulation cases to be used in the rest of this section.

- 4-2 antenna configuration system: Macrocells and femtocells have 4 and 2 antennas per BS, respectively.

- Case 1. Both tiers use SUBF

- Case 2. Both tiers use SDMA

- Case 3. First tier uses SDMA and the other SUBF

- Case 4. First tier uses SUBF and the other SDMA

- 2-1 antenna configuration system: Macrocells and femtocells have 4 and 1 antennas per BS, respectively.

- Case 5. First tier uses SUBF and the other SISO

- Case 6. First tier uses SDMA and the other SISO

- SISO system: Case 7: Both tiers use SISO.

\section{A. Coverage Probability}

We present coverage probabilities for a 4-2 configuration system in Fig. 2 for full SDMA and SUBF cases with $\alpha=4$ and compare them with the SISO case. We observe that when both the tiers perform SUBF, the system achieves the best coverage probability. This is intuitive because transmission from the serving BS is improved due to the beamforming gain while the effective fading power from the interfering links remains the same as the SISO case. Similarly SDMA performs worse than SISO because the mean fading power of interfering links increases and thus causes strong interference while serving link remains the same as the SISO case. The intermediate cases where one tier uses SDMA and the other SUBF lie in-between these two extreme cases.

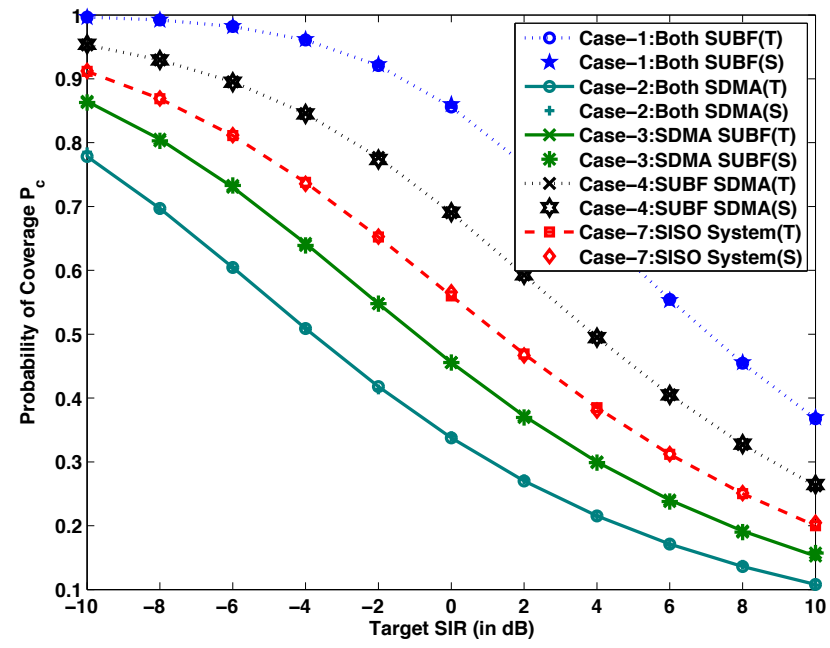

Fig. 2. Coverage probability of a two tier HetNet with $\alpha=4, \lambda=$ $[150,300], P_{1}=5 \cdot P_{2}=50$ for $4-2$ antenna configuration with SUBF and SDMA techniques. The baseline SISO case is also included. T and $\mathrm{S}$ respectively denote theoretical and simulation results. Bias is $\sqrt{\Psi_{j} / \Delta_{j}}$.

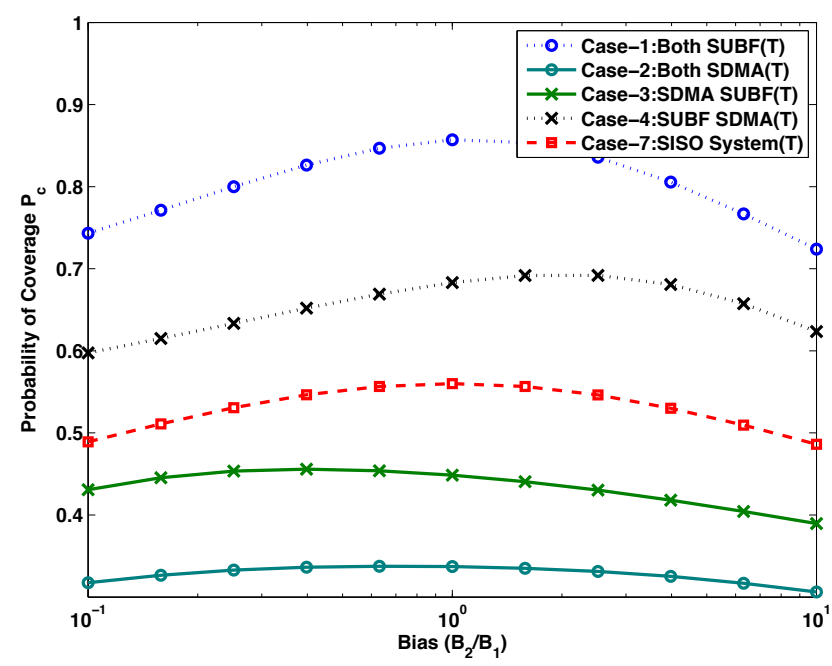

Fig. 3. Coverage probability versus relative bias $B_{2} / B_{1}$ in a two tier HetNet with $\alpha=4, \lambda=[150,300], P_{1}=5 \cdot P_{2}=50$ for SIR target $0 \mathrm{~dB}$ for $4-2$ antenna configuration.

\section{B. Optimal Bias}

As discussed, unlike SISO, the MIMO HetNet requires a bias even for coverage maximization which can be optimized. We plot the variation of the coverage probability versus bias $\left(B_{2} / B_{1}\right)$ in Fig. 3 for target SIR of $0 \mathrm{~dB}$ for different cases of 4-2 antenna configuration. In Fig. 4, we determine the accuracy of modified bias approximation by comparing the bias found from different selection-bias candidate functions and the coverage optimal bias found from simulation for all 7 cases defined earlier in this section. Note that the BS selection based on the highest mean SINR may not always be an optimal approach from coverage maximization perspective. However, from Fig. 4 it is evident that the candidate function (9) is still a reasonable choice. Further, since it depends solely upon the number of antennas at the BS and the number of users served in each resource block, it is easier to implement in practice. 


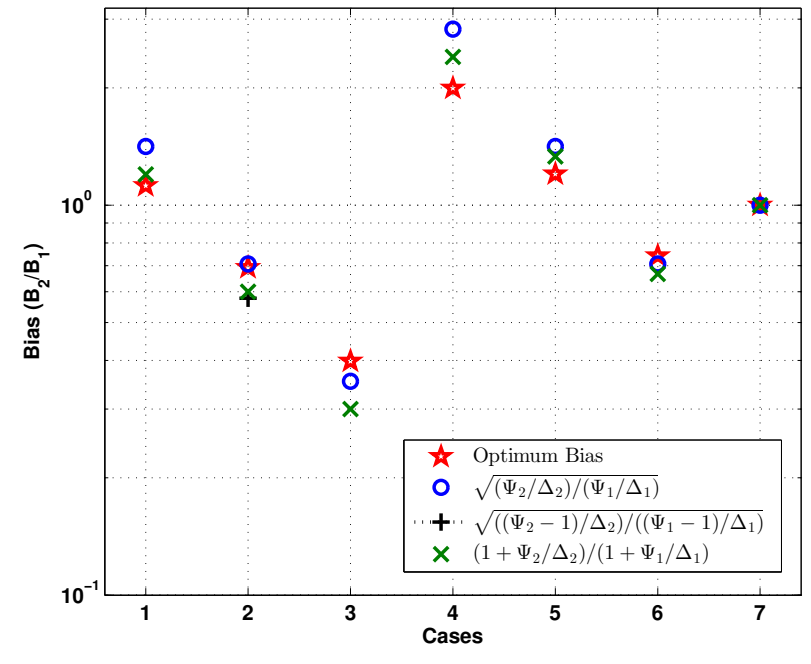

Fig. 4. Comparison of different candidates functions with numerically obtained bias to maximize coverage probability for target SIR $0 \mathrm{~dB}, \lambda=$ $[150,300], P_{1}=5 \cdot P_{2}=50$ for all the 7 simulation cases.

\section{CONCLUSION}

In this paper, we have presented a framework to model and analyze the downlink MIMO HetNet with general cell selection and discussed key differences between MIMO and SISO HetNets. We derived a simple selection-bias based cell association criterion that closely approximates the complex selection rules to maximize mean SINR. For this cell selection rule, we derived exact expression for coverage probability. Future work could include computation of rate coverage and derivation of per-tier bias expression for downlink rate maximization. Another useful direction is to assume multiple antennas at the users and study the impact of this additional degree of freedom on system design, especially in terms of cell selection and load balancing across tiers.

\section{APPENDIX}

\section{A. Proof for Derivatives of the Laplace Transform}

The Laplace transform of noise plus interference $\mathcal{L}_{I}(s)$ can be written as $f(g(s))$ where $f(x)=\exp (x)$ and

$$
g(s)=2 \pi \sum_{j=1}^{K} \lambda_{j} \int_{r_{j}}^{\infty}\left(-1+\frac{1}{\left(1+s P_{j} r^{-\alpha}\right)^{\Psi_{j}}}\right) r \mathrm{~d} r .
$$

Using Faà di Bruno's lemma, $n^{\text {th }}$ derivative can be written as

$\frac{\mathrm{d}^{n} \mathcal{L}_{I}(s)}{\mathrm{d} s^{n}}=\frac{\mathrm{d}^{n} f(g(s))}{\mathrm{d} s^{n}}=\sum_{\bar{m} \in M} C(\bar{m}) f^{1^{T}} \bar{m}(g(s)) \prod_{l=1}^{n}\left(g^{(l)}(s)\right)$

The $l^{\text {th }}$ derivatives of $g(s)$ can be computed as $g^{(l)}(s)$

$$
\begin{aligned}
& =2 \pi \sum_{j=1}^{K} \lambda_{j}(-1)^{l} \frac{\left(\Psi_{j}+l-1\right) !}{\left(\Psi_{j}-1\right) !} P_{j}^{l} \int_{r_{j}}^{\infty} \frac{r^{1-l \alpha}}{\left(1+s P_{j} r^{-\alpha}\right)^{\Psi_{j}+l}} \mathrm{~d} r \\
& \stackrel{(a)}{=} 2 \pi \sum_{j=1}^{K}(-1)^{l} D_{j}(l) P_{j}^{l}\left(s P_{j}\right)^{\frac{2}{\alpha}-l}
\end{aligned}
$$

where $(a)$ can be computed using the transformations $\left(s P_{j}\right)^{-1 / \alpha} r \rightarrow t$ and $1 /\left(1+t^{-\alpha}\right) \rightarrow u$ successively.

\section{B. Proof for Coverage Probability}

The coverage probability, $P_{c}$, can be expressed as

$$
\begin{aligned}
& 2 \pi \lambda_{k} \int_{0}^{\infty} \sum_{n=0}^{\Delta_{k}-1} \frac{1}{n !}\left(T P_{k}^{-1}\right)^{n} . \\
& \sum_{\bar{m} \in M} C(\bar{m}) F(\bar{m}) e^{-\left[\sum_{j=1}^{K} \lambda_{j}\left(T \widehat{P_{j}}\right)^{\frac{2}{\alpha}} \mathcal{C}_{j}\right] x^{2}} x^{2 \sum m_{l}} . \\
& x e^{-\pi \sum_{j=1}^{K} \lambda_{j}\left(\widehat{P_{j} \widehat{\Delta}_{j}} \widehat{B_{j}}\right)^{2 / \alpha} x^{2}} \mathrm{~d} x \\
& =2 \pi \lambda_{k} \sum_{n=0}^{\Delta_{k}-1} \frac{1}{n !}\left(T P_{k}^{-1}\right)^{n} \text {. } \\
& \sum_{\bar{m} \in M} C(\bar{m}) F(\bar{m}) \int_{0}^{\infty} e^{-\left[\sum_{j=1}^{K} \lambda_{j}\left(T \widehat{P}_{j}\right)^{\frac{2}{\alpha}} \mathcal{C}_{j}\right] x^{2}} . \\
& e^{-\left[\pi \sum_{j=1}^{K} \lambda_{j}\left(\widehat{P_{j}} \widehat{\Delta_{j}} \widehat{B_{j}}\right)^{2 / \alpha}\right] x^{2}} x^{1+2 \sum m_{l}} \mathrm{~d} x \\
& \stackrel{(a)}{=} \pi \lambda_{k} \sum_{n=0}^{\Delta_{k}-1} \frac{1}{n !}\left(T P_{k}^{-1}\right)^{n} \text {. } \\
& \sum_{\bar{m} \in M} \frac{C(\bar{m}) F(\bar{m}) \Gamma\left(\sum m_{l}+1\right)}{\left[\sum_{j=1}^{K} \lambda_{j}\left(T_{P_{j}}\right)^{\frac{2}{\alpha}} \mathcal{C}_{j}+\pi \sum_{j=1}^{K} \lambda_{j}\left(\widehat{P_{j}} \widehat{\Delta}_{j} \widehat{B}_{j}\right)^{2 / \alpha}\right]^{\sum m_{l}+1}}
\end{aligned}
$$

where $(a)$ follows from $\int_{0}^{\infty} e^{-a x^{2}} x^{2 n+1}=1 /(a)^{n+1}$.

\section{REFERENCES}

[1] H. S. Dhillon, R. K. Ganti, and J. G. Andrews, "A tractable framework for coverage and outage in heterogeneous cellular networks," in Proc., Information Theory and its Applications (ITA), San Diego, CA, Feb. 2011.

[2] H. S. Dhillon, R. K. Ganti, F. Baccelli, and J. G. Andrews, "Modeling and analysis of K-tier downlink heterogeneous cellular networks," IEEE Journal on Sel. Areas in Commun., vol. 30, no. 3, pp. 550 - 560, Apr. 2012.

[3] H.-S. Jo, Y. J. Sang, P. Xia, and J. G. Andrews, "Heterogeneous cellular networks with flexible cell association: A comprehensive downlink SINR analysis," IEEE Trans. on Wireless Commun., vol. 11, no. 10, pp. 3484 - 3495, Oct. 2012.

[4] P. Madhusudhanan, J. G. Restrepo, Y. Liu, T. X. Brown, and K. Baker, "Downlink performance analysis for a generalized shotgun cellular systems," 2012, available online: arxiv.org/abs/1002.3943.

[5] S. Mukherjee, "Distribution of downlink SINR in heterogeneous cellular networks," IEEE Journal on Sel. Areas in Commun., vol. 30, no. 3, pp. 575 - 585, Apr. 2012.

[6] H. S. Dhillon and J. G. Andrews, "Downlink rate distribution in heterogeneous cellular networks under generalized cell selection," IEEE Wireless Commun. Letters, vol. 3, no. 1, pp. 42 - 45, Feb. 2014.

[7] H. ElSawy, E. Hossain, and M. Haenggi, "Stochastic geometry for modeling, analysis, and design of multi-tier and cognitive cellular wireless networks: A survey," IEEE Commun. Surveys and Tutorials, vol. 15, no. 3, pp. 996 - 1019, 2013.

[8] Q. Li, G. Li, W. Lee, M. Lee, D. Mazzarese, B. Clercks, and Z. Li, "MIMO techniques in WiMax and LTE: a feature overview," IEEE Communications magazine, vol. 48, no. 5, pp. 86-92, May. 2010.

${ }^{l}[9]$ H. S. Dhillon, M. Kountouris, and J. G. Andrews, "Downlink coverage probability in MIMO HetNets," in Proc., IEEE Asilomar, Pacific Grove, CA, Nov. 2012.

[10] — "Downlink MIMO HetNets: Modeling, ordering results and performance analysis," IEEE Trans. on Wireless Commun., vol. 12, no. 10, pp. $5208-5222$, Oct. 2013.

[11] R. W. Heath, Jr., M. Kountouris, and T. Bai, "Modeling heterogeneous network interference using Poisson point processes," IEEE Trans. on Signal Processing, vol. 61, no. 16, pp. 4114 - 4126, Aug. 2013.

[12] D. B. Taylor, H. S. Dhillon, T. D. Novlan, and J. G. Andrews, "Pairwise interaction processes for modeling cellular network topology," in Proc., IEEE Globecom, Anaheim, CA, Dec. 2012.

[13] B. Blaszczyszyn, M. K. Karray, and H.-P. Keeler, "Using Poisson processes to model lattice cellular networks," available online: arxiv.org/abs/1207.7208. 